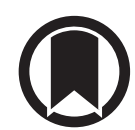

CrossMark

\section{The impact of COVID-19 and the restoration of tuberculosis services in the Western Pacific Region}

\author{
To the Editor:
}

We read with great interest the papers by STOCHINo et al. [1], TADOLIN et al. [2] and Ong et al. [3], which reported issues related to coronavirus disease 2019 (COVID-19) and tuberculosis (TB). The response to COVID-19, especially the lockdown of entire society, is likely to cause severe disruption of TB programmes [4-7]. Reduced TB case notifications have been reported from the top three high TB burden countries worldwide, raising concerns of un-interrupted TB transmission and increased TB mortality, thus posing the threat of derailing achievements made so far [4-7]. Furthermore, the process of post-lockdown restoration of $\mathrm{TB}$ services is important [7]. A slow restoration course may continue decreasing TB case finding as compared to a more rapid restoration time.

To share the experience of national responses to the impact of COVID-19 on TB, the Western Pacific Regional Office of the World Health Organization coordinated an online meeting of the Technical Advisory Group on TB and National TB Programme Managers of the Region on 23-24 June 2020. There were more than 90 participants from 17 countries. Experiences in different countries were presented and strategies to address challenges of TB prevention and care in the era of COVID-19 were discussed.

In China, non-medical interventions, including cancellation of public transportation, prohibition of public gathering and requirement for citizens to stay at home, have successfully brought COVID-19 under control [8-10]. However, these interventions have been associated with more than $20 \%$ reduction in national TB case detection in February 2020 compared with February 2019. A national survey on the impact of COVID-19 on TB found that restriction of traffic has created barriers for patients to access healthcare, difficulties for health providers in tracing presumptive TB cases, difficulties in sputum transportation, difficulties in ensuring TB patients' adherence to treatment, and challenges in visiting patients' households for contact tracing. Staff from national TB programmes, TB laboratories and TB wards have been re-assigned to the fight against COVID-19. Concern regarding COVID-19 transmission has reduced willingness of patients to visit health facilities. In response, online diagnosis and prescription of anti-TB medicines were introduced, independent TB wards were established to meet the need of TB patients, electronic means of treatment support was applied, health education to mitigate panic over COVID-19 was strengthened, procedures for testing for both COVID-19 and TB were developed, and personal protective equipment (PPE) for community health workers was provided.

In Malaysia, border control, restriction of movement, social distancing and self-protection through PPE have resulted in a flattened COVID-19 incidence curve. However, these interventions have also caused a 9\% reduction of TB case detection in January to May 2020 compared with that in 2019. To restore TB services, several new practices have been introduced, including online fast lane appointment for clinical visits, TB screening among COVID-19 cases, drive-through and dropbox for sputum collection and enhancing mobile chest radiography tools to avoid overcrowding in health facilities. Furthermore, physical distancing and use of PPE was advocated. Treatment support has been changed from facility-based to community-based to reduce patient' visits to health facilities. Continuous monitoring of national TB programmes and frequent reminders to implementers helped improve TB case detection and management.

@ERSpublications

While COVID-19 has imposed great challenges to national tuberculosis programmes, it has also created opportunities to develop innovative strategies to ensure continuity of TB services and ultimately to strengthen TB care, prevention and control https://bit.ly/3bHDQx0

Cite this article as: Chiang C-Y, Islam T, Xu C, et al. The impact of COVID-19 and the restoration of tuberculosis services in the Western Pacific Region. Eur Respir J 2020; 56: 2003054 [https://doi.org/ 10.1183/13993003.03054-2020]. 
TABLE 1 Priority for rapid restoration of tuberculosis (TB) care and prevention during the coronavirus disease 2019 (COVID-19) pandemic

\section{Domains Actions of priority}

Case finding - Development of new standards of clinical consultations

- Establishment of affordable online consultations for individuals with presumptive TB

- Strengthening of specimen transportation using innovative approaches

- Development of algorithm for dual TB and COVID-19 testing among individuals with respiratory symptoms

- Application of digital platform and connectivity solutions for rapid delivery of test results

- Strengthening of contact examinations during community quarantine for detection of active TB and management of latent TB infection

Treatment - Innovative approach for enrolment of bacteriologically confirmed TB cases on anti-TB treatment to prevent initial loss to follow-up

- Strengthening of community-based TB treatment services to reduce health facility visits and prevent interruption of treatment

- Scaling-up of digital adherence interventions, such as video directly observed therapy (VOT), short message services and innovative use of mobile applications

- Establishment of digital mechanisms for the management of adverse reactions, especially for patients with rifampicin-resistant TB

Resources - Multi-sectorial approaches for restoration and enhancement of budget and human resources for TB prevention and care

- Mobilisation of community health workers, civil society organisations and community-based organisations to strengthen response to both TB and COVID-19

- Innovative approaches for training of health workers and health education of the community on TB and COVID-19 through digital platforms

In the Philippines, community quarantine began in Manila on 15 March 2020, and subsequently was implemented in most parts of the country. Consequently, active TB case finding was discontinued; visits of symptomatic individuals to health facilities was restricted; priority of health services was shifted to COVID-19; GeneXpert platforms in selected TB culture laboratories were re-assigned to COVID-19 screening; and TB contact tracing was limited. The effect was an immediate decrease of $78.7 \%$ in aggregate weekly TB notification following community quarantine. To ensure continuity of $\mathrm{TB}$ services, the programme issued a memorandum that a 1-month supply of drugs should be given to patients and new standards will be issued through the national TB programme adaptive plan. Screening for TB was subjected to mandated social distancing and infection control procedures. Algorithm of handling specimens for TB testing in the community with ongoing transmission of COVID-19 was developed. Home based TB treatment by using digital adherence technologies has been introduced.

After the presentations of China, Malaysia and the Philippines, participants were divided into three groups to discuss the impact of COVID-19, the opportunities and priorities for TB programmes. Response to COVID-19 has resulted in disruption of TB services and decreased detection of both active TB and latent TB infection in several countries [4]. Priority actions for rapid restoration of TB services from group discussions are summarised in table 1. While COVID-19 has imposed great challenges to national TB programmes, it has also created opportunities to develop innovative strategies to ensure continuity of $\mathrm{TB}$ services and ultimately to strengthen TB care, prevention and control.

Chen-Yuan Chiang ${ }^{1}$, Tauhid Islam ${ }^{2}$, Caihong $\mathrm{Xu}^{3}$, Thilaka Chinnayah ${ }^{4}$, Anna Marie Celina Garfin ${ }^{5}$, Kalpeshsinh Rahevar ${ }^{2}$ and Mario Raviglione ${ }^{6}$

${ }^{1}$ International Union Against Tuberculosis and Lung Disease, Paris, France. ${ }^{2}$ End TB and Leprosy Unit, Division of Programmes for Disease Control, WHO Regional Office for the Western Pacific, Manila, Philippines. ${ }^{3}$ National Center for Tuberculosis Control and Prevention, Chinese Center for Disease Control and Prevention, Beijing, China. ${ }^{4}$ Tuberculosis and Leprosy Control Sector, Disease Control Division, Ministry of Health, Putrajaya, Malaysia. ${ }^{5}$ Department of Health, Disease Prevention and Control Bureau, Manila, Philippines. ${ }^{6}$ Centre for Multidisciplinary Research in Health Science (MACH), University of Milan, Milan, Italy.

Correspondence: Chen-Yuan Chiang, 68, Boulevard Saint-Michel, 75006 Paris, France. E-mail: cychiang@theunion.org

Received: 7 Aug 2020 | Accepted after revision: 3 Sept 2020

Conflict of interest: None declared.

\section{References}

1 Stochino C, Villa S, Zucchi P, et al. Clinical characteristics of COVID-19 and active tuberculosis co-infection in an Italian reference hospital. Eur Respir J 2020; 56: 2001708.

2 Tadolini M, Codecasa LR, García-García J-M, et al. Active tuberculosis, sequelae and COVID-19 co-infection: first cohort of 49 cases. Eur Respir J 2020; 56: 2001398. 
3 Ong CWM, Migliori GB, Raviglione M, et al. Epidemic and pandemic viral infections: impact on tuberculosis and the lung. A consensus by the World Association for Infectious Diseases and Immunological Disorders (WAidid), Global Tuberculosis Network (GTN) and members of ESCMID Study Group for Mycobacterial Infections (ESGMYC). Eur Respir J 2020; 56: 2001727.

4 Migliori GB, Thong PM, Akkerman O, et al. Worldwide effects of coronavirus disease pandemic on tuberculosis services, January-April 2020. Emerg Infect Dis 2020; in press: [https://doi.org/10.3201/eid2611.203163].

5 Pang Y, Liu Y, Du J, et al. Impact of COVID-19 on tuberculosis control in China. Int J Tuberc Lung Dis 2020; 24: 545-547.

6 Glaziou P. Predicted impact of the COVID-19 pandemic on global tuberculosis deaths in 2020. medRxiv 2020; preprint [https://doi.org/10.1101/2020.04.28.20079582].

7 Cilloni L, Fu H, Vesga JF, et al. The potential impact of the COVID-19 pandemic on tuberculosis: a modelling analysis. medRxiv 2020; preprint [https://doi.org/10.1101/2020.05.16.20104075].

8 Pan A, Liu L, Wang C, et al. Association of public health interventions with the epidemiology of the COVID-19 outbreak in Wuhan, China. JAMA 2020; 323: 1915-1923.

9 Tian $\mathrm{H}$, Liu Y, Li Y, et al. An investigation of transmission control measures during the first 50 days of the COVID-19 epidemic in China. Science 2020; 368: 638-642.

10 Zhang J, Litvinova M, Liang Y, et al. Changes in contact patterns shape the dynamics of the COVID-19 outbreak in China. Science 2020; 368: 1481-1486.

The content of this work is copyright of the authors or their employers. Design and branding are copyright (c)ERS 2020

This version is distributed under the terms of the Creative Commons Attribution Non-Commercial Licence 4.0. 